\title{
Denuncias éticas de médicos colegiados en Perú, 1991-2010: frecuencia y naturaleza
}

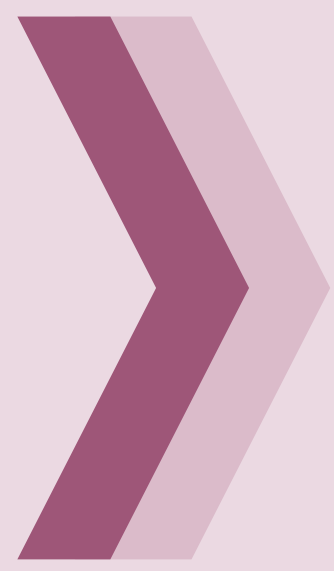

\author{
Ethical demands of \\ collegiated physicians \\ in peru 1991-2010: its \\ frequency and nature
}

\section{Cecilia Sogi-Uematzu, Maximiliano Cárdenas-Díaz y Salomón Zavala-Sarrio}

Universidad Nacional Mayor de San Marcos

\section{RESUMEN}

Introducción. El Colegio Médico del Perú (CMP), creado por la Ley N. 15173 del año 1964, vela por el cumplimiento de las normas éticas de la profesión, específicamente, a través de su Comité de vigilancia ética y deontológica. En casos de infracción al código, el Consejo Nacional o el Consejo Regional respectivo pone fin al procedimiento disciplinario, imponiendo sanción o declarando la absolución del médico investigado, mediante una resolución. Овлетіvo. Describir la frecuencia y naturaleza de las denuncias éticas contenidas en las resoluciones del Consejo Nacional, en el período 1991-2010. Material y métodos. Se accedió a las resoluciones del Consejo Nacional. Se elaboró una ficha de recolección de datos; y se utilizaron el Excel para la base de datos y el SPSS versión 15 para el análisis de frecuencia. La naturaleza de las faltas éticas se estableció con base en el Código de ética y deontología del CMP (versiones 1970 y 2000). Resultados. En el primer decenio, las 84 resoluciones (24,8\%) contenian 157 infracciones al código, las más frecuentes fueron en el título relación médico paciente (35,7\%). En el segundo decenio, las 253 resoluciones (75,2\%) contenian 388 infracciones, las más frecuentes fueron en el título derechos del paciente (55\%). Conclusıones. La frecuencia de las denuncias éticas se triplicó entre los dos decenios. La naturaleza de las trasgresiones al código fueron las mismas en los dos decenios, aunque su frecuencia varía por diferencias entre sus dos versiones (1970 y 2000).

Palabras clave: Ética, códigos de ética, ética médica, mala conducta profesional, ética profesional.

\begin{abstract}
InTRODUction. The Medical College of Peru, created by law N. 15173 of 1964 , is in charge of watching the professional conduct of physicians through its ethics and deontology committee. In case of the infraction to the code, the National Council or Regional Council finishes the disciplinary process imposing sanction or dismissing the complaint through a resolution. Oвjectives. To describe the frequency and nature of claims cases against doctors disciplined through the resolutions of the National Council. Material and METHODS. The National Council's resolutions were acceded. A format for recording case characteristics was developed. Excel for data base and SPSS 15 for statistical analysis were used. The codes of 1970 and 2000 were used in order to describe the nature of infractions. REsults. In the first decade of study, the 84 resolutions $(24,8 \%)$ contained 157 infractions to the code, the most frequent was at title medical patient relationship (35,7\%). In the second decade, the 253 resolutions $(75,2 \%)$ contained 388 infractions, the most frequent was at title patient's rights (55\%). Conclusions. The frequency of ethical demands increased three times between the two decades. The nature of infractions were the same although its frequency varied due to differences between the codes of 1970 and 2000 .
\end{abstract}

KEY WORDs. Ethics, codes of ethics, ethics, medical, professional misconduct, ethics, professional. 


\section{INTRODUCCIÓN}

En occidente, los inicios de la medicina se remontan a 2500 años, en Grecia, con los hipocráticos. En ese entonces, eran los propios médicos los que se daban para sí normas de conducta basados en el principio de beneficencia $y$, sobre todo, en la máxima primun non nocere. Posteriormente, en el curso de la historia, se instituyen organismos encargados de velar por la conducta profesional del médico. Así, en la edad media surge el Protomedicato, desarrollado en su máxima expresión en España, en el siglo XV, como "tribunal encargado de autorizar, controlar y aplicar medidas punitivas en el ejercicio de la profesión médica y de organismo asesor del gobierno en todo lo concerniente a salubridad". ${ }^{1}$

En Inglaterra, en 1421, fueron los propios médicos quienes solicitaron el control del estado en la definición de sus funciones. Para ello presentaron al parlamento la primera petición registrada de este tipo en Inglaterra y, muy probablemente, por primera vez en Europa. ${ }^{2}$ Luego, en 1858, el parlamento inglés decretó el Medical Act y con ello se crea el Consejo General de Educación y Registro Médico del Reino Unido, actualmente conocido como General Medical Council (GMC), encargado, entre otras atribuciones, del registro de médicos hábiles y de regular la conducta profesional. $^{3}$

De otro lado, según MacKenzie, el concepto de medicina como profesión fue creado por dos médicos eticistas, el escocés Scott John Gregory (1724-1773) y el inglés Thomas Percival (17401804). Antes de Gregory, dice el autor, que los médicos utilizaban el término profesión para diferenciarse ellos mismos de los cirujanos, boticarios y otros competidores, no formados en universidades, como empíricos de bajo nivel. Los escritos de Gregory, basados en el principio de simpatía de Hume, introducen un cambio vital en énfasis y orientación, y privilegian al paciente. Señala que los autores citados fueron los primeros médicos en emplear el término paciente, en lugar de enfermo. Los principios (códigos) de Gregory, enfatizan las competencias científica y clínica, la precaución contra el interés personal del médico e introduce la idea de la medicina como deber público (opuesto al gremio de mercaderes, lo que era en aquellos tiempos). Con base en las ideas de su predecesor, Percival introduce el término ética médica aplicada a la conducta del médico en hospitales y asilos, la etiqueta profesional, autor del libro Medical Ethics, publicado en $1803 .{ }^{4}$

Hoy en día, el ejercicio de la medicina está regulado en la mayoría de los países y al respecto resulta ilustrativo el estudio que llevó a cabo el GMC en diez países, Egipto, Alemania, Grecia, India, Italia, Nigeria, Pakistán, Polonia, Sudáfrica y España. Las conclusiones del informe final de este estudio son los siguientes:

$\Delta$ El registro con un organismo médico regulador, antes de que el médico inicie su ejercicio, es un requerimiento formal en todos los países, con excepción de un pequeño número de regiones autónomas de España.

- La necesidad de una licencia para ejercer es relativamente similar en los diez países.

- La revalidación, similar a la del Reino Unido, es infrecuente en los países en estudio.

- Todos los países tienen un código de ética médica, que tiene un patrón relativamente homogéneo.

- Resulta difícil discernir en qué medida los pacientes y el público (así como los propios médicos) son conscientes del código de ética médica.

- Todos los países tienen un procedimiento disciplinario, pero existen variaciones substanciales en cuanto al ente responsable.

- Los tipos de sanciones son llamada de atención, amonestación, suspensión, expulsión, difusión pública y multa.

- Todos tienen organismos para regular y asegurar la calidad de la educación médica, pero el tipo de organismo responsable varía entre los países.

- Las aproximaciones al desarrollo profesional continuado varía ampliamente entre los países, pero la atención que los reguladores les prestan a esto es cada vez mayor. ${ }^{5}$ 


\section{Conducta profesional y su regulación}

En todos los países existen instituciones encargadas de velar que la conducta del médico se desenvuelva dentro de los cánones establecidos en sus respectivos códigos de ética. Así, en el Reino Unido, el GMC mantiene el registro de médicos hábiles y regula la conducta profesional con base en el libro azul, Professional conduct and discipline: fitness to practice, el mismo que describe el proceso que sigue las denuncias contra médicos, organizado en cuatro instancias: 1) staff del GMC; 2) seleccionador inicial; 3) comité de procedimiento preliminar; $y, 4)$ comité de conducta profesional. Cada etapa del proceso es descrita por Smith, en tres artículos publicados en British Medical Journal, en 1989.6-8

\section{Estados Unidos}

En 1847, se funda la American Medical Association (AMA), como organización dedicada a establecer normas uniformes para la formación profesional, la capacitación y la conducta de los médicos. En esa fecha se aprobó, por unanimidad, su primer código de ética profesional que articula los valores perdurables de la medicina como profesión, con los que se comprometen los médicos, individual y colectivamente. ${ }^{9}$ Este código resulta un hito especialmente importante, por ser la primera vez en la historia que una comunidad médica nacional se daba para sí, por decisión autónoma, un código de conducta. El código constituye un documento orientador, una guía para promover la conducta de los médicos a título de recomendaciones que, explícitamente como lo recalcan los preámbulos de todas las ediciones, no tiene carácter vinculante, es decir, no sanciona. En su lugar, los médicos estadounidenses son disciplinados por las juntas médicas estatales (state medical boards) y las sanciones van desde amonestación hasta revocación de la licencia para el ejercicio profesional. La junta médica de California, por ejemplo, clasifica sus motivos para la acción disciplinaria en nueve categorías mayores: negligencia, prescripción médica impropia, ejercicio médico sin licencia, inmoralidad sexual, enfermedad mental, actos que ponen en riesgo al paciente por consumo de sustancias del médico, fraude, condena por un delito y conducta no profesional. ${ }^{10}$

\section{Argentina}

El Colegio de la provincia de Buenos Aires, creado por Ley en 1958, de afiliación obligatoria para los médicos de la localidad, vela por el cumplimiento de su código de ética y tiene potestad sancionadora. La Confederación de Entidades Médicas Colegiadas (Confemeco) coordina los colegios provinciales. En el sistema de salud argentino, Tobar señala que: "se producen más de 350000 consultas y más de 8000 internaciones hospitalarias por día, de ellas en promedio solo dos generan demanda de mala praxis. Entre médicos y odontólogos, ejercen unos 138000 profesionales, de los cuales 10000 (7,2\%) han sido denunciados por supuesta mala praxis en los últimos treinta años. Del total de las demandas, 32\% recaen en el médico y $68 \%$, en instituciones médicas, incluidas obras sociales y empresas de medicina prepaga. La mitad de tales demandas no prosperan y de las que lo hacen, la mitad genera condena y la otra mitad, conciliaciones. Dos de cada diez médicos cuentan con esquemas de protección, ya sean seguros o fondos solidarios. ${ }^{11}$

\section{Brasil}

El Consejo Federal de Medicina (CFM), creado por ley en 1945 , supervisa y regula la práctica médica a través del registro de médicos hábiles; dicta y aplica el código de ética imponiendo sanciones, normado en 2001.

Fujita (1992) llevó a cabo un estudio retrospectivo de las denuncias presentadas al Consejo Regional de Medicina de Goiás, entre 2000 y 2006. Halló que $62 \%$ de las quejas alegaron incompetencia profesional y relación médicopaciente inadecuado; y $50 \%$ de tales quejas fueron contra las especialidades de cirugía plástica y ortopedia. ${ }^{12}$ Otra encuesta, del Consejo Regional de Medicina de Sao Paulo, halló que las diez especialidades que recibieron más quejas fueron ginecología y obstetricia, pediatría, ortopedia y traumatología, medicina del trabajo, oftalmología, cirugía plástica, cardiología, psiquiatría, gastroenterología y enfermedades infecciosas. La mayoría de tales quejas estaban relacionadas con negligencia, incompetencia o imprudencia, mala atención, vínculos con la industria, comportamiento impropio, entre otras. ${ }^{13}$ 


\section{Chile}

El Colegio Médico de Chile, creado por ley en 1948, vela por: "el perfeccionamiento, la protección económica y social y la supervigilancia de la profesión de médico-cirujano". ${ }^{14}$ En el año 2005, se reconoce la facultad de los colegios profesionales de procesar demandas por inconducta ética de sus miembros y para ello se dispuso tribunales. El Colegio organizó un Fondo de Defensa Legal Médica (Falme), encargada de la defensa, en los procesos penales y civiles contra médicos por presunción de negligencia. En 2004, el promedio mensual de juicios por negligencia médica fue de 87 mientras que en 2000 era 45 y en 1998, 16,3. ${ }^{15}$

\section{Colombia}

El estado asume directamente la regulación ética de los médicos a través del tribunal ético profesional. Los miembros son designados por el Ministerio de Salud a propuesta de la Federación Médica, Academia de Medicina y Facultades de Medicina. El Tribunal ético profesional de Bogotá, instalado en 1994, hasta 2002, atendió quejas que involucraron 1070 pacientes, de los cuales $29,7 \%$ fallecieron. ${ }^{16}$

\section{Ecuador}

La Federación Médica ecuatoriana es reconocida por ley como la instancia que lleva el registro del médico, a través de los colegios provinciales, y el control ético, a través de los tribunales de honor. Existen presiones para que se incluya en el código penal a la negligencia médica como un delito explícitamente tipificado. ${ }^{17}$

\section{Uruguay}

Recientemente, en el año 2009, se crea el Colegio Médico de Uruguay, como institución de afiliación obligatoria para el ejercicio profesional, con el cometido de "garantizar al médico y a la comunidad el ejercicio de la medicina dentro del marco deontológico establecido". No existen estudios sobre la magnitud de las denuncias éticas contra médicos pero existen continuas referencias, en los medios de comunicación y las revistas profesionales, al incremento de demandas judiciales civiles y penales presentadas por pacientes insatisfechos o dañados en el curso de la atención médica así como los montos de indemnización. Esto llevó al Sindicato Médico de Uruguay a la creación de un servicio de asistencia integral profesional, encargada de la defensa legal y la cobertura de las indemnizaciones. ${ }^{18}$

\section{Venezuela}

La Federación Médica Venezolana (FMV), fundada en 1945 , es reconocida por ley en 1982, como entidad representativa de la profesión, lleva el registro de médicos a través de los colegios regionales; elabora y aprueba el código de ética; $y$, procesa las denuncias éticas en los tribunales disciplinarios (nacional y regionales). En el año 2011, el gobierno reformó esta ley y extendió las atribuciones hasta entonces exclusivas de la FMV a otras organizaciones gremiales. ${ }^{19}$

\section{Perú}

La Ley N. 15173 del año 1964, crea el Colegio Médico del Perú (CMP) y su modificatoria, Decreto Ley 17239 , contiene dos artículos vinculados a la regulación ética y legal del ejercicio de la medicina. El artículo 5, que establece los fines del Colegio, en su acápite dice: "a) Velar por que el ejercicio de la profesión médica se cumpla de acuerdo con las normas deontológicas contenidas en el código de ética profesional que el Colegio dicte"; y el artículo 11 señala: "En los casos de infracción al Código de Ética Profesional o de las Resoluciones emanadas por los Consejo Nacionales cometidas por los profesionales colegiados, el Colegio le hará conocer su extrañeza y según sea el caso lo expulsará de su seno. La suspensión no podrá ser mayor de un año y en caso de reincidencia, no mayor de dos años, las suspensiones y expulsiones que emanen de los Consejos Regionales deberán ser ratificadas por el Consejo Nacional para poder entrar en vigor. En el caso de condena judicial que imponga inhabilitación, el Colegio procederá a la suspensión por el tiempo que dure la condena. El reglamento establecerá las condiciones necesarias para la rehabilitación y señalará el procedimiento a seguir en todos los casos de aplicación de sanciones y las atribuciones que correspondan a cada una de las instancias". ${ }^{20}$

En el curso de la existencia del CMP, ha habido tres revisiones del código primigenio de 1970, en los años 1997, 2000 y 2007. En el segundo código, que entró en vigencia en octubre de 2000 , es 
descrito por Mendoza como: "el resultado de un amplio e intenso debate que se inició cuatro años antes de su aprobación y en el que participó no únicamente la profesión médica sino también la sociedad civil, a través de representantes de instituciones públicas y privadas relacionadas con la salud. Proceder de otro modo hubiera significado violentar la esencia misma de la ética que, siguiendo a Gracia, debe ser civil o secular, pluralista, autónoma y racional. Como fruto de este consenso se incorporaron los principios de la bioética, los derechos de los pacientes, la prohibición expresa de participar en actos de lesa humanidad, la defensa de la competencia ética del Colegio Médico sobre cualquier otra jurisdicción y la responsabilidad social compartida del médico, la sociedad y el Estado para garantizar el derecho a la salud de todas las personas. Pero, como el impulso ético no puede quedarse fijado, una nueva revisión hizo posible la dación del actual Código que, aprobado en 2007, entró en vigencia el año 2008. El nuevo Código desarrolla más ampliamente lo concerniente a los derechos de los pacientes y los conceptos de la bioética". ${ }^{21}$

En el país, solo hallamos dos estudios sobre procedimiento disciplinario que ejerce el CMP, uno el de Mendoza, ya citado,, ${ }^{21}$ y el otro de Véliz, publicado en $2000 .{ }^{22}$

\section{Objetivo del estudio}

Describir la frecuencia y naturaleza de las denuncias éticas examinadas por el Comité de ética y deontología del Colegio Médico del Perú, en el período 1991-2010.

\section{MATERIAL Y MÉTODOS}

\section{Material}

Resolución final del Consejo Nacional del CMP motivada por la denuncia ética del médico colegiado, correspondientes al período 1991-2010. Este documento contiene datos de nombre de quien entabla la demanda; nombre, número de colegiatura y especialidad (si la tuviera) del médico implicado; el motivo de la denuncia; y, finalmente, la sanción que establece el Consejo.

En el CMP, el Comité de Vigilancia ética y deontológica, tanto del Consejo Nacional como de los 27
Consejos regionales, es el encargado de difundir y vigilar el cumplimiento de la Ley y del Estatuto, Reglamento y Código de ética y deontología del CMP. Si el Comité mencionado hallara indicios de trasgresión a las normas, solicitará la apertura del procedimiento disciplinario al Comité de Asuntos contenciosos y procedimientos disciplinarios. El Reglamento, en su capítulo II. Del procedimiento ético disciplinario, describe, paso a paso, cómo terminan las denuncias en una resolución (Art. 145: El Consejo regional y en su caso el Consejo Nacional, con el informe emitido por el Comité de Asuntos contenciosos y procedimientos disciplinarios, emitirá la resolución que pone fin al procedimiento, imponiendo sanción o declarando la absolución de los colegiados sujetos a investigación). Adicionalmente, el reglamento contempla la impugnación de la mencionada resolución (Art. 147.6: Una vez emitido el dictamen a que se refiere el párrafo precedente, el Consejo Nacional deberá pronunciarse mediante resolución debidamente motivada por la confirmatoria o revocatoria de la resolución impugnada..... ${ }^{23}$

El material del presente estudio corresponde al pronunciamiento del Consejo Nacional sobre la resolución impugnada de los veintisiete consejos regionales. Por consiguiente, nuestros datos no se corresponden con la totalidad de las denuncias atendidas por el Colegio sino a una fracción de ella, aquellas que merecieron la resolución definitiva del Consejo Nacional.

\section{Instrumento}

Se elaboró una ficha de recolección de datos de las denuncias éticas, que incluía fecha y localidad de la denuncia, quien denuncia, especialidad y número de colegiatura del médico, naturaleza de la falta y la sanción.

\section{Procedimiento}

Se solicitó autorización para acceder a la resolución final emitida por el Consejo Nacional del CMP, correspondiente al período 1991-2010. Se ubicó un total de 377 resoluciones, de ellas se separaron $40(10,6 \%)$ por incompletas (no incluían motivo de la denuncia ni la sanción). Así, la muestra del presente estudio fue de 337 resoluciones. Se mantuvo la confidencialidad de los datos. 
No todas las resoluciones revisadas consignan el número del(os) artículo(s) a los que faltó el médico denunciado. En estos casos se procedió con la prueba de confiabilidad interevaluador, es decir, dos investigadores, en forma independiente, asignaron el número de artículo del código, correspondiente a la descripción de la falta. Luego, discutieron las diferencias para llegar a un consenso.

La naturaleza de las denuncias éticas se separaron en aquellas correspondientes al primer período (1991-2000) y las del segundo (2001-2010) porque se utilizaron dos versiones del Código de ética y deontología, para el primero la del año 1970 y para el segundo la del año 2000. En seis resoluciones, las faltas éticas se tipificaron utilizando la última versión del código (2007), pero para fines del presente estudio fueron convertidas de acuerdo al código del año 2000. Las diferencias entre la primera y la segunda versión del código son descritas por Mendoza. ${ }^{22}$

\section{Análisis de datos}

Se elaboró una base de datos en Excel y se utilizó el SPSS versión 15 para análisis de frecuencias. Se determinó la frecuencia de faltas a las normas éticas y para ella se siguió el orden establecido en artículos y títulos del código.

\section{RESULTADOS}

Se identificaron 337 resoluciones finales del Consejo Nacional del CMP entre 1991-2010. Corresponden al primer decenio, $84(24,8 \%)$ y al segundo, $253(75,2 \%)$, es decir, la frecuencia de las denuncias aumentó tres veces entre los dos decenios de estudio. El mayor número de denuncias correspondan a Lima, $86,0 \%$ en el primer decenio y $76,7 \%$ en el segundo. Sin embargo, la frecuencia de las resoluciones de otras regiones del país aumentó comparativamente, de $14 \%$ al $23,3 \%$ en el segundo decenio.

\section{Frecuencia de las denuncias por su naturaleza}

La naturaleza de las denuncias que trasgreden las normas éticas se agruparon de acuerdo a títulos del Código de ética y deontología del CMP, en sus versiones de los años 1970 y 2000. Tabla 1.

\begin{tabular}{lrr}
$\begin{array}{l}\text { Tabla 1. Orden de frecuencia de faltas éticas. } \\
\text { Código de ética y deontología } 1970 \\
\end{array}$ & $n=157$ & $\%$ \\
\hline Relación médico paciente & 56 & 35,7 \\
$\Delta$ Publicidad & 29 & 18,5 \\
$\Delta$ Relaciones entre médicos & 24 & 15,3 \\
$\Delta$ Trabajo médico & 18 & 11,5 \\
$\Delta$ Especialistas & 8 & 5,1 \\
$\Delta$ Obligaciones pecuniarias & 5 & 3,2 \\
$\Delta$ Relaciones de los médicos con familiares & 4 & 2,5 \\
$\Delta$ Certificados & 4 & 2,5 \\
$\Delta$ Prescripción de medicamentos & 3 & 1,9 \\
\hline Otros & 6 & 3,8 \\
\hline
\end{tabular}

* Un caso en cada una de las siguientes faltas éticas: injerto, trasplante de órganos, aborto, secreto profesional, honorarios, ejercicio ilegal de la medicina, sanciones.

La Tabla 1, correspondiente al primer decenio de estudio, es sobre un total de 157, porque el número total de faltas al código fluctuó entre uno $(63,0 \%)$, dos $(18,0 \%)$, tres $(9,6 \%)$ y cuatro o más $(6,0 \%)$ por denuncia. Una denuncia contenía 21 faltas al código. Se observa que las infracciones más frecuentes fueron en el título Relación médico paciente $(35,7 \%)$; seguidas de las de Publicidad $(18,5 \%$ ) y de las de Relaciones entre médicos (15,3\%).

La Tabla 2, correspondiente al decenio 2001-2010, es sobre un total de 388 , porque el número total de faltas al código por cada denuncia fluctuó entre uno $(67,6 \%)$, dos $(17,0 \%)$, tres $(7,5 \%)$ y cuatro a más (7,5\%). Las infracciones más frecuentes fueron en el título Derechos del paciente ( $55 \%$ ), seguida de las de Relaciones entre médicos (13,4\%). Si se agrupan como documentos al certificado, la historia clínica y otros registros, suman $42(10,8 \%)$, de modo que las faltas del título III Documentos médicos serían la tercera en orden de frecuencia.

Un hallazgo negativo que cabe destacar es la ausencia de faltas en los títulos VII, el médico y la docencia, y VIII, el médico y la investigación en el código de 1970, y la poca frecuencia de las faltas en actividades de investigación y docencia en el código de 2000 (0,3\%). 


\begin{tabular}{lrr}
$\begin{array}{l}\text { Tabla 2. Orden de frecuencia de faltas éticas. } \\
\text { Código de ética y deontología } 2000 \\
\end{array} \quad \mathrm{n}=388$ & $\%$ \\
\hline Derechos de los pacientes & 213 & 54,9 \\
$\Delta$ Relaciones entre médicos & 52 & 13,4 \\
$\Delta$ Certificados médicos & 25 & 6,4 \\
$\Delta$ Trabajo médico & 23 & 5,9 \\
$\Delta$ Historias clínicas y otros registros médicos & 17 & 4,4 \\
$\Delta$ Disposiciones generales & 14 & 3,6 \\
$\Delta$ Médicos legistas y auditores & 10 & 2,6 \\
$\Delta$ Secreto profesional & 9 & 2,3 \\
$\Delta$ Competencia y perfeccionamiento & 7 & 1,8 \\
$\Delta$ Publicidad & 6 & 1,5 \\
$\Delta$ Prescripción médica & 5 & 1,3 \\
$\Delta$ Relaciones con otros profesionales & 5 & 1,3 \\
$\Delta$ Otros* & 2 & 0,6 \\
\hline
\end{tabular}

*Actos contra la humanidad; docencia e investigación.

Diferencias en las versiones del

Código de ética y deontología de 1970 y de 2000

Las faltas relacionadas con publicidad, que fueron la segunda causa de denuncia en el primer decenio, se ubicaron entre las menos frecuentes $(1,5 \%)$ en el segundo. Cabe mencionar que en la versión 2000 del código, a diferencia de su predecesora solo contiene tres artículos. Asimismo, en el código del 2000 no existe lo que corresponde al ejercicio ilegal de la medicina (artículo 171 en el código de 1970).

\section{Casos judiciales}

La base de datos del presente estudio incluyó resoluciones del Consejo Nacional del CMP correspondientes a casos judiciales, 6 en el primer decenio y 18 en el segundo. La naturaleza de estos casos fueron las siguientes:

- Homicidio culposo (delito contra la vida, el cuerpo y la salud).

- Atentado contra la administración pública peculado en agravio del Estado.

$\Delta$ Delito contra la libertad en su modalidad de violación sexual.
- Delitos contra la familia, estado civil, alteración o supresión de la filiación del menor; el Estado por delito contra la vida y la salud.

\section{DISCUSIÓN}

El CMP, en cumplimiento de sus funciones, lleva a cabo actividades de vigilancia ética y deontológica de sus miembros, de un lado, a través de procedimientos ético disciplinarios de los médicos involucrados en denuncias; $y$, de otro, alienta los procesos de acreditación de facultades de medicina, la recertificación profesional y otras actividades educativas. El presente estudio examina la primera de las actividades, la de vigilancia ética del Colegio. Se identificaron 337 denuncias éticas contenidas en las resoluciones finales del Consejo Nacional del CMP, desde 1991 hasta 2010.

\section{Limitaciones del estudio}

Respecto al número de denuncias por faltas éticas, nuestros datos constituyen solo una parte de la totalidad de denuncias atendidas por el CMP. Muchas de ellas son resueltas a nivel de los consejos regionales y solo aquellas que han sido impugnadas a este nivel merecen finalmente una resolución del Consejo Nacional. Determinar la magnitud de la totalidad de denuncias sobrepasa los esfuerzos individuales y requiere de la participación activa de los 27 Consejos Regionales, para el seguimiento del proceso ético disciplinario. No obstante, se considera que los hallazgos del presente estudio reflejan lo que viene ocurriendo respecto a las denuncias por faltas éticas de médicos colegiados en el país.

Frecuencia de las denuncias éticas 1991-2010

Un hallazgo notorio del presente estudio es el aumento en la frecuencia de denuncias éticas en el CMP, en el período de estudio, tres veces entre los dos decenios. Esta preocupante tendencia debe ser motivo de posteriores estudios analíticos orientados a identificar los factores asociados a tal incremento.

De otro lado, en las últimas décadas, un fenómeno que se observa en el país es el aumento en el número de médicos a la par que el de la población peruana y su mayor cobertura de salud. En el 
año 1970, cuando se inició el registro nacional de médicos en el país, el número de colegiación asignado fue hasta el 7208 . A fines de 1990, el número era de 24058, es decir, entre 1970 y 1990, el número de nuevos médicos se había incrementado en 16850 . A fines de la década de 1990, el número de colegiatura era 24058 y, para fines del 2010, era 57871 , es decir, el número de nuevos médicos se había incrementado en 28 210, en el período 1991-2010. Paralelamente, el número de facultades de medicina, de 24 en la década de 1990 pasó a 34 a la fecha. Al respecto, Bazán señala que este aumento: "no solo es debido a lo que permite la autonomía universitaria, sino fundamentalmente a la Ley de Promoción de la inversión en educación (DL N. ${ }^{\circ} 882$ de noviembre de 1996), que en su artículo 2 establece que: "toda persona natural o jurídica tiene el derecho a la libre iniciativa privada para realizar actividades en educación, derecho que permite fundar, promover, conducir y gestionar instituciones educativas particulares con o sin finalidad lucrativa. Sobre la base de esta Ley, varias universidades actúan como empresas privadas y se encuentran fuera del alcance de las regulaciones educativas poniendo el lucro por encima de la calidad de la educación y de la formación médica". ${ }^{24}$

Entre los pocos estudios nacionales sobre el tema de la vigilancia ética y deontológica, se halla el de Véliz, publicado en el año 2000, que corrobora el hallazgo del aumento en la frecuencia de denuncias. Véliz, en 148 denuncias, $10 \%$ de un total de 1500 expedientes correspondientes al período 1980-1999, presentadas al Comité de vigilancia ética y deontológica del Consejo Regional III del CMP, observó una tendencia creciente de las demandas, aunque no precisa su magnitud. ${ }^{22}$

Adicionalmente a lo mencionado, otro factor relacionado con el aumento en la frecuencia de faltas éticas sería la calidad de la educación médica en el Perú. Un estudio sobre la educación ética en el país halló que la enseñanza de la materia en las escuelas de medicina, a nivel nacional, es heterogénea en cuanto a objetivos, contenidos y material de lectura. Sin embargo, existen similitudes en cuanto al poco espacio que se asigna a su enseñanza, de $1 \%$ a $2 \%$ del total de créditos académicos que debe completar el alumno para graduarse de médicos; la enseñanza es en aula y no en campo clínico; y no se enseña ética durante el internado. ${ }^{25}$ Otro estudio, en 159 internos de medicina de una universidad nacional de la ciudad capital, halló necesidades de formación en tres tópicos éticos: 1) veracidad en la información al paciente y a la familia; 2) consentimiento informado; 3) relación médico paciente, relación con colegas y otros profesionales de la salud; asimismo, en temas específicos tales como limitación del esfuerzo terapéutico y ética del examen de partes íntimas. ${ }^{26}$

\section{Naturaleza de las denuncias éticas}

En el primer decenio de estudio, las infracciones más frecuentes fueron en los títulos relación médico paciente $(35,7 \%)$, publicidad $(18,5 \%)$ y relación entre médicos (15,3\%). En el segundo decenio, las infracciones más frecuentes fueron en los títulos derechos del paciente $(55 \%)$ y relaciones entre médicos $(13,4 \%)$.

Nuestros hallazgos son similares a los estudios nacionales de Véliz y Mendoza. Véliz halló que $40 \%$ de las denuncias tenían que ver en gran medida con la relación médico paciente; $27 \%$ con problemas relacionados con medios masivos de comunicación; $16 \%$ con dificultades de relación entre médicos; y $12 \%$ con casos judiciales. ${ }^{22}$ Mendoza examinó los procedimientos éticodisciplinarios evaluados por el Consejo Nacional entre 2004 y 2007. Tenían que ver directamente con el acto médico y, por consiguiente, con la relación médico paciente, 75 denuncias; $y$, con problemas de relación interpersonal de los propios médicos, 47 denuncias. Señala, asimismo que entre mayo y junio de 1999, en los 37 juzgados de Lima hubo 190 médicos procesados, 59 por homicidio culposo, 14 por lesiones culposas y 4 por exposición o abandono de personas en peligro, lo que representaba 1,2 \% de los médicos que por entonces ejercían en Lima. No considera los juzgados del Ministerio Público, los de la Región Callao y del cono norte de Lima. ${ }^{21}$

En Brasil, Bitencourt et al, revisaron casos de proceso ético profesional del Consejo Regional de Bahía (Cremeb) entre 2000 y 2004. De los 372 médicos procesados en ese período, 159 (42,7\%) fueron por negligencia médica (artículo 29 Practicar actos profesionales perjudiciales para 
el paciente, que podría ser caracterizados como incompetentes, imprudentes, o negligentes). La incompetencia se identificó en 107 (67,3\%) de las denuncias; la imprudencia, en 37 (23,3\%); la negligencia, en $14(8,8 \%){ }^{27}$

En Colombia, el Tribunal de ética médica del Valle del Cauca revisó casos juzgados durante el quinquenio 2000-2004 y halló que se atendieron 308 quejas. De estas, 219 fueron objeto de investigación, por presunta violación a las normas éticas, por lo cual se inició el respectivo proceso ético disciplinario; resultado del mismo fueron sancionados 75 profesionales (34,2\%) con amonestaciones, censuras y suspensión del ejercicio profesional. De estas sanciones, $57,3 \%$ correspondieron a violaciones de normas vinculadas al capítulo I de la Ley 23: de las relaciones del médico con el paciente. ${ }^{16}$

En Australia y Nueva Zelandia, el estudio de Elkin et al. (2011) halló que las lesiones iatrogénicas ocurrieron en $9 \%$ y la muerte del paciente en $8 \%$ de los casos. Respecto al tipo de inmoralidad, el primero fue sexual ( $24 \%$ de los casos), que ocurrió exclusivamente con médicos varones y la más frecuente fue las relaciones sexuales con pacientes y entre otros contactos sexuales impropios (examen innecesario, tocamiento de partes íntimas); prescripción ilegal 0 no ética ( $21 \%)$; atención médica inadecuada (20\%), que incluye problemas de tratamiento y errores diagnósticos. Otras faltas fueron quebrantar las condiciones del registro; las relacionadas con el certificado médico o la historia clínica; no obtención del consentimiento informado; delitos no relacionados con pacientes, entre otras. ${ }^{28}$

El código de ética médica prescribe las normas de conducta que debe observar el profesional en su relación con el paciente. Tradicionalmente, el precepto que guía la norma es el principio de beneficencia y sobre todo la máxima primun non nocere. En el contexto de la relación médico paciente, el código contiene normas que resguardan al profesional de incurrir en error y negligencia: respetar la vida y actuar con la máxima diligencia; y, su infracción está sujeta a sanción. Respecto a la mayor frecuencia de trasgresiones en los títulos derechos del paciente (1970) y derechos del paciente (2000), se considera que estas se relacionan con eventos adversos, error y negligencia médica. En las últimas dos décadas, los estudios sobre error médico -su contexto, causas, consecuencias y costos- se han extendido en amplitud y profundidad. Hurwitz y Sheikh sostienen que: "Los errores, vistos previamente como un fenómeno residual y evento desafortunado al margen de la atención de salud, se les considera, hoy en día, como parte integrante del ejercicio de la medicina... los errores médicos adquieren presencia institucionalizada en revistas cuando el New England Journal of Medicine, en 1984, publica un dramático error que daña seriamente a una mujer, seguida de Lancet, en 1999, que hace un llamado a los médicos para que compartan, por escrito, sus experiencias con tales eventos. ${ }^{29}$

\section{Eventos adversos, error y negligencia médica}

En Estados Unidos, en 1984, se llevó a cabo el Harvard Medical Practice Study (HMPS), estudio epidemiológico de eventos adversos y negligencia en pacientes hospitalizados en el estado de Nueva York. Los autores del estudio definen eventos adversos como una lesión ocasionada por intervención médica (más que por la enfermedad subyacente), que prolonga la estancia hospitalaria, produce incapacidad al momento del alta, o ambos. La negligencia es definida como la falla en alcanzar el estándar de práctica, que se espera razonablemente de un médico, en la atención de un paciente dado. ${ }^{30}$ El HMPS halló una incidencia de eventos adversos de 3,7\%, y de estos, $27,6 \%$ fueron debidos a negligencia; $70,5 \%$ de tales eventos dio lugar a incapacidad que duró menos de seis meses, 2,6\% condujeron a incapacidad permanente, y $13,6 \%$, a muerte. ${ }^{31}$

El HMPS es uno de los pocos que estima la tasa de lesiones intrahospitalarias a nivel de población general compilados por Weiler et al. Así, en 2,6 millones de pacientes dados de alta de hospitales neoyorkinos, en 1984, cerca de 99000 sufrieron lesiones discapacitantes y de estos 56000 fueron deterioro leve con recuperación al mes. Otros 13500 sufrieron deterioro moderado con recuperación en seis meses. Más de $70 \%$ de los eventos adversos identificados causó incapacidad relativamente breve. Sin embargo, hubo un número de sujetos que sufrieron lesiones de mayor gravedad: más de 3800 eventos produjeron deterioro permanente, con incapacidad mayor de 
$50 \%$; y otros, 2500 incapacidad grave o total, como resultado del tratamiento. Más dramático fue aun que 13400 pacientes neoyorkinos murieron en 1984 como resultado del tratamiento médico. ${ }^{32}$

En Canadá, como parte de un esfuerzo para la creación del Instituto de Seguridad del Paciente Canadiense, se llevó a cabo un estudio sobre eventos adversos. En este se estimó que 7,5\% de los pacientes admitidos a un hospital de atención de agudos, durante el año 2000, experimentaron uno o más eventos adversos. De estos, $36,9 \%$ se juzgaron como casos potencialmente previsibles. ${ }^{33}$ En América Latina, se llevó a cabo un estudio de prevalencia de eventos adversos en pacientes hospitalizados (IBEAS, 1998). En este participaron seis países: España. México, Perú, Argentina, Costa
Rica y Colombia. El IBEAS halló que la prevalencia global de pacientes con algún evento adverso (EA) fue $10,5 \%$; y la de EA, $11,9 \%$. Los EA detectados estaban relacionados con los cuidados, $13,3 \%$; uso de medicación, 8,2\%; infección nosocomial, $37,1 \%$; algún procedimiento, $28,7 \%$; y diagnóstico, $6,2 \%$. Los tipos de eventos más frecuentes que hacen un total de $36,2 \%$ fueron las neumonías nosocomiales $(9,4 \%)$, las infecciones de heridas quirúrgicas $(8,2 \%)$, las úlceras por presión (7,2\%), otras complicaciones relacionadas con intervención quirúrgica o procedimiento $(6,4 \%)$ y sepsis o bacteriemia (5\%). De estos eventos, $62,9 \%$ de los eventos aumentaron el tiempo de hospitalización a una media de 16,1 días y 18,2\% causaron reingreso. ${ }^{34}$

\section{Agradecimiento}

Estudio de investigación con asignación y con incentivos 2012, Universidad Nacional Mayor de San Marcos. Este estudio no hubiera sido posible sin el apoyo del Colegio Médico del Perú.

\section{REFERENCIAS BIBLIOGRÁFICAS}

I. Laval E.El protomedicato en el desarrollo de la salubridad en Chile durante la Colonia y la República. [revista en la Internet]. [accesado 10 de mayo de 2014] Rev Chil Infectol. 2003;20(Suppl): I I7-I I7. Disponible en: http://www.scielo.cl/scielo.php?script=sci_ arttext\&pid=S07|6-10182003020200043\&lng=es. http://dx.doi. org/l0.4067/S07|6-10182003020200043.

2. Raach J. English medical licensing in the early Seventeenth Century.Yale J Biol Med. 1944; 16(4):267-288

3. General Medical Council [Sede Internet]: 2014. England. [accesado 10 de mayo de 2014]; 20( Suppl): I 17-I I7. Disponible en: www.gmc-uk.org

4. MacKenzie CR. HSS J. 2007 September; 2007; 3: 222-227

5. General Medical Council. International_Comparison_of_Ten Medical_Regulatory_Systems_final_report [Sede internet]. United Kingdom: RANND Corporation. 2009. [accesado 10 de mayo de 2014]. Disponible en: International_Comparison_of Ten_Medical_Regulatory_Systems_final_report.pdf_25404378. pdf

6. Smith R. Discipline I: the hordes at the gates. BMJ. 1989;298: I502-I505.

7. Smith R. Discipline II: the preliminary screener - a powerful gatekeeper. BMJ. 1989;298: I 569-I57I.

8. Smith R. Discipline III: the final stages. BMJ. 1989;298:1632-I634.

9. American Medical Association. AMA's Code of Medical Ethics/ Ethics Timeline: 1847 to 1940: AMA Code of Medical Ethics written and published. [Sede Internet]. 2014. USA. [accesado 10 de mayo de 2014]. Disponible en: http://www.ama-assn. org/ama/pub/about-ama/our-history/history-ama-ethics/ethicstimeline-1847-1940.page?
10. Papadakis MA, Hodgson CS, Teherani A, Kohatsu ND. Un professional behavior in medical school is associated with subsequent disciplinary action by a state medical board. Acad Med. 2004;79:244-249.

II. Tobar F. Economía de la mala praxis médica en Argentina Revista Médicos [revista en Internet]. 2013 julio [accesado 10 de mayo de 2014]. Disponible en: http://www.revistamedicos.com.ar/ economia_m_praxis.htm

12. Fujita R. Denúncias por erro médico en Goiás. Rev Assoc Med Bras. 1992;55(3):283-289.

13. O Conselho Regional de Medicina do Estado de São Paulo. Guia da Relação Médico-Paciente. [sede Internet]. Brasil. Conselho Regional de Medicina do Estado de São Paulo. 200I [accesado 10 de mayo de 2014]. Disponible en: http://www.cremesp.org. $\mathrm{br} /$ ?siteAcao $=$ Publicacoes\&acao $=$ detalhes\&cod_publicacao $=4$

14. Ley 9.263. Crea el Colegio Médico de Chile. Diario Oficial 10 de diciembre de 1948. Ley 9.263. [Internet]. Diario Oficial 10 de diciembre de 1948. [accesado 10 de mayo de 2014]. Disponible en: http://www.i-derecho.cl/shnoti.asp?noticia=1058

15. Sagredo F.Auge y juicios por negligencia contra médicos: camino hacia una medicina defensiva. Rev Vida Médica. 2004;56(2):6-9. Colegio Médico de Chile. [revista en Internet]. [accesado 10 de mayo de 2014]. Disponible en: http://www.colegiomedico. $\mathrm{cl} /$ Portals/0/files/biblioteca/publicaciones/revista_medica/ rvm56_2.pdf

16. Amorocho N, Guzmán N. Por qué demandan a los médicos ante el Tribunal de Ética Médica en el departamento del Valle del Cauca.Tribuna Ética Médica. 2008: I I I 9 [revista en Internet]. [accesado 10 de mayo de 2014]. Disponible en: http://www. 
temvalle.org/revista/revista-tribuna-etica-medica $\% 20$ vol-3\%20 $\mathrm{N}-\mathrm{I} . \mathrm{pdf}$

17. Federación Médica Ecuatoriana. Los médicos anuncian que harán una vigilia por la paz en Plaza Grande [sede Internet]. Ecuador. FME. 20I4. [accesado 10 de mayo de 20I4]. Disponible en: http:// federacionmedicaecuatoriana.info/index.php/2013-0I-10-04-2432/gremiales/26I-los-medicos-anuncian-que-haran-una-vigiliaen-la-plaza-grande

18. Franca O. Hacia el primer código uruguayo de ética médica. Rev Med Uruguay. 1989;5:3-10.

19. Cubero-Castillo. La Ética Médica en Venezuela (189519|8). [Revista en Internet]. Bitácora-e Revista Electrónica Latinoamericana de Estudios Sociales, Históricos y Culturales de la Ciencia y la Tecnología. 2012;1:4-17. [accesado 10 de mayo de 2014]. Disponible en: http://www.saber.ula.ve/ bitstream//23456789/35555/I/articulol.pdf

20. Ley N. ${ }^{\circ}$ 15173. Creando el Colegio Médico del Perú como entidad autónoma de Derecho Público. Colegio Médico del Perú. [Página en Internet]. [accesado 10 de mayo de 2014]. Disponible en: http://www.cmp.org.pe/doc_norm/ley_creacion_cmp.pdf

21. Mendoza A. Ética y medicina: la experiencia del Colegio Médico del Perú. Rev Peru Med Exp Salud Pública. 201 I;28(4):670-675.

22. Veliz J. Características de 148 denuncias presentadas al Comité de Vigilancia Ética y Deontológica del Consejo Regional III del Colegio Médico del Perú.Acta Med Peruana. 2000;20(I):40-47.

23. Colegio Médico del Perú. Reglamento. [sede Internet]. Colegio Médico del Perú. Perú. 2014. [accesado 10 de mayo de 20I4]. Disponible en: http://www.cmp.org.pe/doc_norm/ reglamentocmp.pdf

24. Bazán C. El acto médico. En el acto médico. Cuadernos de debate en salud. Lima: Fondo Editorial Comunicacional, Colegio Médico del Perú; 20II. pp. 16-24.

25. Cárdenas M, Sogi C. Enseñanza de la ética en las escuelas de medicina peruanas: un estudio de sílabos.An Fac Med. 20 13;74(2): I07-II5.
26. Zavala S, Sogi C. Evaluación de la formación ética de internos de medicina 2003. Universidad Nacional Mayor de San Marcos. An Fac Med. 2006;68(I):5-18.

27. Bintencourt, Almir Galvão Vieira, et al. Análise do erro médico em processos ético-profissionais: implicações na educação médica. Rev Bras Educ Med. 2007;31(3):223-228. [revista en Internet]. [accesado 10 de mayo de 2014]. Disponible en: http:// www.scielo.br/pdf/rbem/v3 In3/04.pdf

28. Elkin KJ, Spittal MJ, Elkin DJ, Studdert DM. Doctors disciplined for professional misconduct in Australia and New Zealand 2000 2009. MJA. 20II;194: 452-456.

29. Hurwitz B, Sheik A. Medical fallibility. Cultural recognition and representation. JJR Soc Med. 2009: 102: I8I-185.

30. Leape LL, Brennan TA, Laird NM, Laethers AG, Localio R, Barnes BA, et al. The nature of adverse events in hospitalized patients. Results of the Medical Practice Study II. N Engl J Med. 199I;324;377-384.

3I. Brennan TA, Leape LL, Laird NM, Hébert L, Localio R, Lawthers AG, et al. Incidence of adverse events and negligence in hospitalized patients. Results of the Harvard Medical Practice Study I. N Engl J Med. I991;324:370-376.

32. Weiler PC, Hiatt HH, Newhouse JP, Johnson WG, Brennan TA, Leape LL. Medical injury, malpractice litigation and patient compensation. In: A measure of malpractice. 3rd edition. Cambridge: Harvard University Press; 1993.

33. Baker GR, Norton PG, Flintoft V, Blais R, Brown A, Cox J, et al. The Canadian Adverse Events Study: the incidence of adverse events among hospitalized patients in Canada. CMAJ. 2004; I70(II):1678-I686.

34. Aranaz Andrés JM, Aibar Remón C, Cárdenas García F, et al. Estudio IBEAS Prevalencia de efectos adversos en hospitales de Latinoamérica. España. Informes, Estudios e Investigación: Ministerio de Sanidad y Política Social. 2009 [Informe en Internet]. [accesado 10 de mayo de 2014]. Disponible en: http://190.12.79.236/calidad/images/pdf/publicaciones/IBEAS\%20 informe.pdf

\section{Correspondencia}

Dr. Salomón Zavala-Sarrio szavalas@gmail.com

\section{Conflictos de interés}

Los autores declaran no tener conflictos de interés durante el planteamiento, ejecución de la investigación y la elaboración del artículo para su publicación.

Fecha de recepción: 9 de mayo de 2014

Fecha de aceptación: 26 de mayo de 2014 